\title{
Mine Layout Design and Medium-Term Prediction of Seismicity
}

\author{
S.M. Spottiswoode CSIR Miningtek, South Africa
}

\begin{abstract}
Mine layout designs under rockburst conditions aim to control the incidence of seismicity and rockbursts. Design decisions are based on previous experience, numerical simulations of proposed mining and assessment of the seismic history in the area, and on grade. The numerical simulations and seismic analysis are generally undertaken separately and some formal or informal weighting system is used when decisions are made.

This paper introduces a methodology in which the spatial distribution of future seismicity is estimated based on past seismicity and past and future elastic energy release. It is shown that this combined approach may more accurately estimate the impending seismicity than using either modelling or previous seismicity on their own.
\end{abstract}

\section{INTRODUCTION}

\subsection{Prediction of Seismicity}

Mine design is based on the assumption that the rock behaviour to planned mining can be predicted with some degree of confidence so that the ore body can be mined safely and at a profit, with a low probability of nasty surprises that will put the operation at risk. This involves living with any seismicity that may occur. This requires some idea of the likely seismicity, in other words, we are called upon to predict the likely incidence of seismicity, the resulting strong ground motion and rockburst damage. It is convenient to divide the time scale of prediction into short, medium and long-term.

Many have seen short-term prediction as the most desirable goal, in which an assured warning is given within hours or minutes prior to large damaging events occurring. The focus on this work has been on measuring short-term changes in the character of seismicity. In common with earthquake prediction, short-term prediction has met with very limited success in South African gold mines (Spottiswoode and de Beer, 1999).

Long-term prediction is needed to decide how best to mine a new block of ground. This is especially true for green-field mines, but is also necessary for long-term expansion of existing operations. Numerical simulations, supplemented by a strong dose of engineering experience, are used for long-term prediction. These long-term predictions are aimed at wide-scale view of likely seismicity.

When attempting to do medium-term prediction, we have better opportunities of using seismic data together with numerical modelling to estimate the amount of seismicity that will be expected in small regions of a mine, say less than $100 \mathrm{~m}$ in extent, over time periods of the order of one month. This fits in well with operational planning.

\subsection{Medium-Term Seismic Prediction}

Rockbursts from mining-induced seismicity pose the most severe hazard in deep-level mining. While use of appropriate support will contain rockburst damage, proper layout design and sequencing is also necessary to reduce seismicity and the resulting strong ground motion in the workings. In the case of deep-level tabular mining, such as in the South African deeplevel gold mines, mine layout design is aimed at reducing the overall seismicity using the now well-known criteria of Energy Release Rate (ERR), Excess Shear Stress and Average Pillar Stress (Ryder and Jager, 2002).

ERR is the criterion most widely used to control span in the relatively unfaulted Carletonville mining region. From about 1970 onwards, strike-parallel pillars were most commonly used to satisfy the ERR criteria set by several mines (e.g. van Antwerpen and Spengler, 1982). In recent years, mines are usually achieving this by leaving regional support pillars aligned on dip (e.g. Handley et al., 2000 and Klokow et al., 2003). Dip pillars offer the advantage that geological features are generally more easily left behind within a skin of bracket pillars than is the case of strike parallel pillars. This increases the extraction ratio and reduces the need to mine through dykes or close to faults.

The level of anticipated seismicity may be estimated in a number of ways. In the ideal case, long-term stability of mines would be assured if enough were known about the rockmass before mining took place. For many reasons, this ideal is never realised and an on-going stability assessment is necessary, based on improved knowledge of the geological structures and the actual response of the rockmass. Furthermore, there are still no simulation programs that can solve the "full" problem of brittle failure resulting from mining of rock around even the most ideal hypothetical mine.

These design criteria are all based on numerical modelling and are sensitive to small changes in rockmass properties and stresses. Seismic data are used on a less detailed basis, but do provide a more direct measure of the response of the rock to mining. Consideration of modelling and seismic data in mine design is generally considered as two entirely different approaches. Only recently have ways been proposed to combine these approaches into a single analysis and design methodology. For the first time, an entire session was devoted to the topic of "Integration of Modelling and Monitoring" at the previous RaSiM conference near Johannesburg, South Africa in 2001.

The use of seismicity to estimate rock strength was studied by Wiles et al. (2001), Beck and Brady (2001) and by Côté et al. (2001). Lachenicht et al. (2001) presented detailed quantitative analysis of seismicity resulting from fault slip. Hoffmann et al. (2001) and Spottiswoode (2004) compared the spatial 
and temporal distribution of observed seismicity to the amount expected from stress or energy changes within the elastic rockmass.

The work of Spottiswoode (2001 and 2004) consisted of back-analyses aimed at understanding the relationships between modelled and observed deformations. In this paper, a more direct and challenging approach to the integration problem is introduced. Both data sets are used to estimate the future seismicity in time and space.

The ultimate objective of this approach is to develop a computer program that will use mine seismic data and modelling to allow a mine to quickly estimate the amount of seismicity that will be likely to follow different mining option. This will assist in deciding between different mining options.

This paper describes my recently developed procedures to predict impending seismicity on a scale of months and $\sim 100 \mathrm{~m}$, implemented in a computer program MinSInt (Mining Seismicity Integrator). Although the results are not sufficiently convincing to be implemented, they are encouraging. Certain conclusions are drawn that will hopefully lead to better results and perhaps procedures that can be implemented.

\section{APPROACHES TO DESIGNING MINE LAYOUTS}

Three approaches to designing macro mine layouts may be used:

\subsection{Numerical Modelling Only}

As mentioned above, this is the normal procedure for macro layout design. A generic approach is initially used to ensure that the layout satisfies the set criteria. In the dip pillar situation, raise spacing, spans and pillar sizes are set. The pillar positions are then adjusted to avoid mining the larger geological discontinuities, but usually without reducing pillar widths. More ground is commonly left behind than was originally planned (e.g. Klokow et al., 2003).

\subsection{Past Seismicity Only}

The hazard posed by tectonic earthquakes may be judged in terms of the likely incidence of strong ground motion. A definition of seismic hazard in mines has been proposed by Kijko et al. (1998) and is based on the probability of events above certain magnitudes occurring within a certain time period.

This hazard definition assumes that the future rate of seismicity will be the same as the past rate of seismicity. This might be true for a large production area, but will, in general, not be true locally as the mining and geological conditions continually change. An immediate improvement in accuracy will be expected if corrections are made for the area to be mined, as is described in this paper.

\subsection{Integration of Modelling with Seismicity}

The "ultimate" integration model will consist of an advanced numerical model that will continually adapt according to the actual response of the rockmass to mining.

In this paper a simple approach to the numerical modelling is described in which only the Energy Release associated with the change in elastic strain energy with mining is considered.

These three approaches are expanded into simple mathematical terms in the next section and then tested in the case study analysed in the following section.

\section{A METHODOLOGY FOR ESTIMATING FUTURE SEISMICITY}

As discussed by Spottiswoode (2004), seismic data needs special processing before it can be compared in spatial detail with modelled results. In particular, the "strength" of each seismic event is distributed into the same square grids that are used for the numerical simulations and are mined at the time of each event.

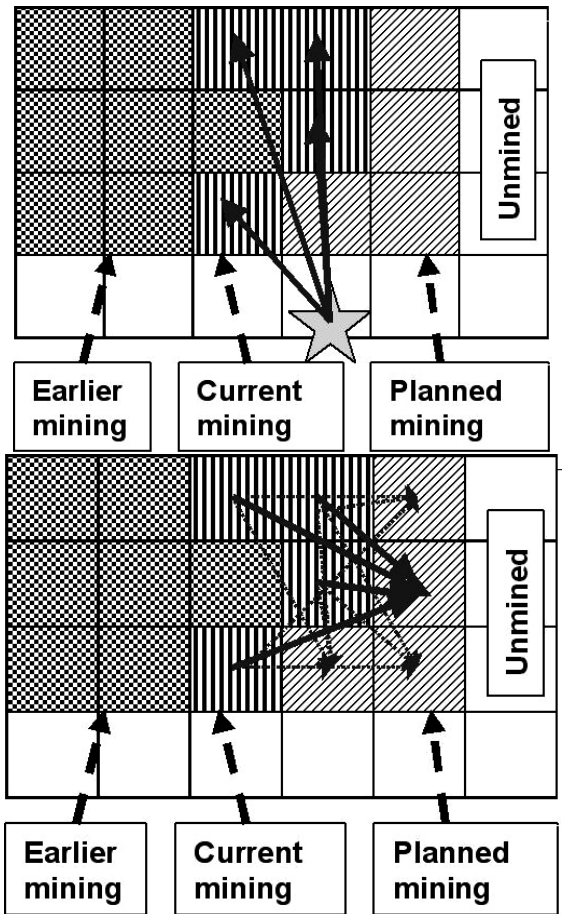

FIG. 1 A sketch illustrating (a) distribution of a seismic event onto the grid squares that are currently being mined, followed by (b) distribution of values in currently mined areas onto planned mining areas

This comparison methodology has been considerably extended in this study to include spatial comparisons between past and future modelling and seismicity on an onreef square grid. This method has two steps (see Figure 1):

1. The location accuracy of mine seismic events in South African gold mines is typically tens of metres, whereas the stress peak on faces and abutments is only several metres wide. We postulate that it is necessary to shift each seismic event onto the face. This is achieved here by distributing each event onto areas of mining with a smoothing function, where the function is set to zero where no energy release is expected. A further smoothing function is applied to emulate the source dimension of each event. This has the effect of spreading the seismic deformation of bigger events over a bigger area than smaller events. Smoothing functions are shown in Figure 2 and their application described in more detail below.

2. One-to-one spatial correspondence and quantitative comparisons between past and future data is obtained by shifted past modelling and seismicity on to areas of planned mining, using the same modified Gaussian function. Seismicity is measured in terms of the parameters listed in Table 1 . The values at each element for which mining is planned are based on a minimum number of mined elements and seismic events, 20 elements and a weighted total of 20 events in the simulations that follow. 
(a)

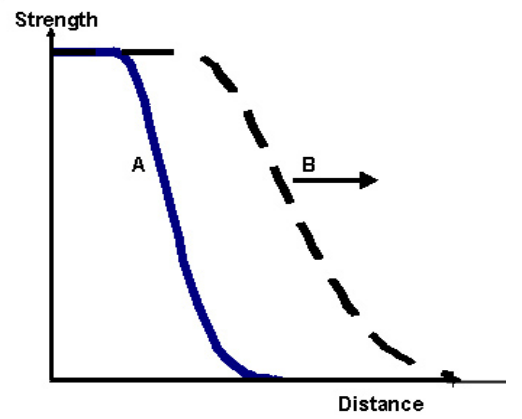

(b)

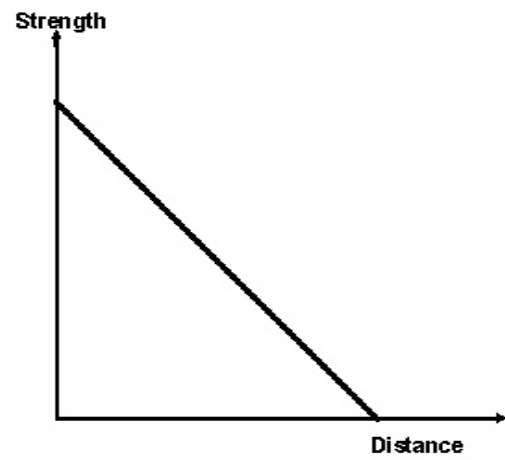

FIG. 2 Smoothing functions. Each is circularly symmetrical. (a) "A" accounts for location error. Expanding functions "A" through "B" or beyond accounts for a region of influence that contains a sufficient number of events and grid elements. (b) Conical function to account for event size

Square grids have been used for many years for modelling the elastic deformations around tabular excavations (Ryder and Jager, 2002).

In this methodology, one set of layers is defined for each time step, with each set consisting of combinations of mining and seismicity data. Each array is used to describe some property in three dimensions, two in space and one in time.

Seismicity can be quantification in various ways (e.g. Table 1). The measure that relates best to the total amount of deformation is seismic moment, expressed as $\Sigma \mathrm{M}_{0^{\prime}}$ the summed seismic moment. However, as a few large events account for most of the total, a simple count of the number of recorded events in any area and time will provide a more even statistical base.

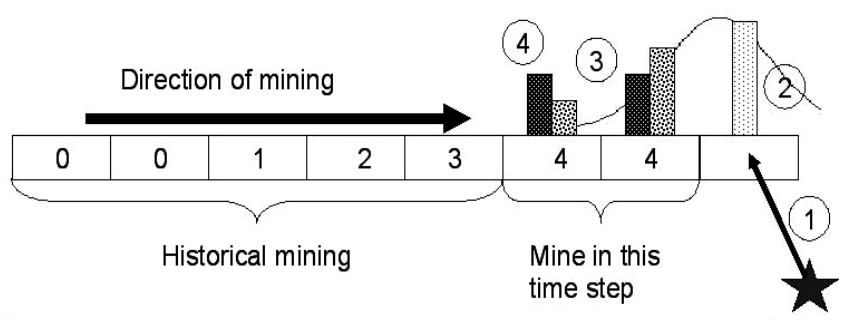

1) Move seismic event to nearest on-reef grid point

2) Give it an appropriate "strength"

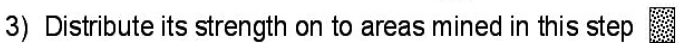

4) Further spread its strength to simulate source size

Operations 3) \& 4) keep the event aligned with mining in step \#4

FIG. 3 A sketch illustrating the process of attributing a seismic event to the area currently being mined. The process is described in the text
TABLE 1 Three different ways in which the seismicity is quantified

\begin{tabular}{lll}
\hline Symbol & Unit & Description \\
\hline no. & 1 & $\begin{array}{l}\text { Number of events: each event is given } \\
\text { equal weight. }\end{array}$ \\
rad_tau & MPa-m & $\begin{array}{l}\text { Source radius times stress drop: a } \\
\text { measure of damage potential } \\
\text { (Spottiswoode 2001). } \\
\text { Mo }\end{array}$ \\
GN-m & $\begin{array}{l}\text { Seismic moment: a measure of source } \\
\text { "strength" }\end{array}$ \\
\hline
\end{tabular}

In this section, the approaches sketched above are expanded into mathematical terms, using the following definitions, where modelled and seismic data are expressed as two-dimensional arrays of values projected onto reef. These definitions were developed especially for this study. It expected that they will change somewhat as this methodology is developed further.
A: Area mined, $\mathrm{m}^{2}$
E: Energy release when mined, J
S: Seismicity, units as per Table 1
G: Modified Gaussian function (Figure 2a)
$\mathrm{C}$ : Conical function (Figure $2 \mathrm{~b}$ )

Two types of smoothing functions are used here:

Modified Gaussian functions (Figure 2a) are used to "correct" the seismic location errors by attributing each event to the nearest areas of mining that may be considered to be the most reasonable source location. These functions are also used to reduce the effect of small sample statistics, while quantifying seismicity and modelled energy release.

Conical functions (Figure $2 b$ ) are used to spread seismic events according the expected source size, as listed in the mine's seismicity catalogue. The conical function is similar in shape to the asperity profile preferred by Lachenicht et al. (2001).

Each of the arrays in the previous list can be modified in various ways and will be indicated by the following superscripts:

C: Current value at each element

M:Current value spatially migrated to the next step.

P: Predicted value in the next step

A: Actual value in the next step.

TABLE 2 Symbols used for array operations and types

\begin{tabular}{ll}
\hline Symbol & Description \\
\hline$\sim$ & Smoothed array \\
$*$ & $\begin{array}{l}\text { Convolution operator } \\
{ }^{*} G\end{array}$ \\
$*^{*} G\|A\|$ & $\begin{array}{l}\text { Smoothes using a modified Gaussian function } \\
\text { the area mined at this step }\end{array}$ \\
\hline$* G\|E\|$ & $\begin{array}{l}\text { Smoothes using a Gaussian function, weighted } \\
\text { according to the energy released in each element }\end{array}$ \\
\hline$* C\|E\|$ & $\begin{array}{l}\text { Smoothes using a Conical source function, } \\
\text { weighted according to the energy released in each } \\
\text { element }\end{array}$ \\
\hline
\end{tabular}


Using these symbols and conventions, Figures $1 \mathrm{a}$ and 3 can be written as:

$$
\widetilde{S}^{C}=\left(S^{C} * G\left\|E^{C}\right\|\right) * C\left\|E^{C}\right\|
$$

Seismicity will be predicted using the three methods described above and the predicted seismicity $\widetilde{S}^{P}$ will be compare in the case study to the actual observed seismicity $\widetilde{S}^{A}$, where $\widetilde{S}^{A}$ is defined in a similar manner to equation [1].

\subsection{Numerical Modelling Only}

The ideal numerical modelling will predict the amount of seismicity, directly. However, if we allow our model to be incorrect by some factor that can be estimated from previous seismicity and modelling in the general area, then we can estimate future seismicity from

$$
S^{P}=E^{P} \times\left(\sum S^{C} / \sum E^{C}\right)
$$

However, this is very demanding on the accuracy of local detail in the numerical model. A more realistic formulation is:

$$
\widetilde{S}^{P}=\widetilde{E}^{P} \times\left(\sum S^{C} / \sum E^{C}\right)
$$

Where the predicted energy release is smoothed over itself as follows:

$$
\widetilde{E}^{P}=E^{P *} G\left\|A^{P}\right\|
$$

This equation is easy to apply because it is not necessary to reallocate values from past mined areas on to planned mined areas.

\subsection{Previous Seismicity Only}

If we assume that the current rate of seismicity per area mined will persist into the future, we can write:

$$
\widetilde{S}^{P}=\widetilde{S}^{M}
$$

where spatial migration takes place through

$$
\widetilde{S}^{M}=\widetilde{S}^{C} * G\left\|E^{P}\right\|
$$

Geological discontinuities have a strong influence on seismicity. The correct practice is to keep an angle of more than $30^{\circ}$ between the face and such features. This results in a concentrated area of excess seismicity following these features as the face advances. The meaning of "current" mining and seismicity in equation [6] is then broadened to include "nearby" mining and seismicity when selecting seismic data. The extent of influence is expanded from each element to be mined until a sufficient number of previous mined elements and seismic events are included. In this study, 20 previous elements and events were considered sufficient. As previously mentioned, the counting of previous events was based on their distributed values; in effect most previously mined elements contained fractional contributions from many events.

\subsection{Integration of Seismicity and Modelling}

We may also assume that the amount of seismicity changes by the change in the elastic strain energy released. This can be expressed as:

$$
\widetilde{S}^{P}=\widetilde{S}^{M} \times \widetilde{E}^{P} / \widetilde{E}^{M}
$$

This simply means that future seismic hazard is expected to be equal to past seismic hazard times the ratio of future ERR to past ERR.

\section{CASE STUDY}

Our study area on No 5E shaft, Driefontein Gold Mine covers an area of about $1000 \mathrm{~m}$ by $1400 \mathrm{~m}$ in extent in which 7 raises were wholly or partially mined. The mine plan was digitised from 1999 to 2002 using the program MinPlan from the MinSim2000 suite of programs. The mine plan was drawn at quarterly intervals for face positions from 1999 to August 2002 and then every month until December 2002. The MinPlan program creates files that contain the pattern of mining over a range of time steps as digitized off the mine plan. The "size" of the simulations was 128 by 128 square elements, each $11 \mathrm{~m}$ on a side. These files were then used as input for program MINF (Spottiswoode, 2001) which then solves for the elastic convergence, stress and elastic energy release.

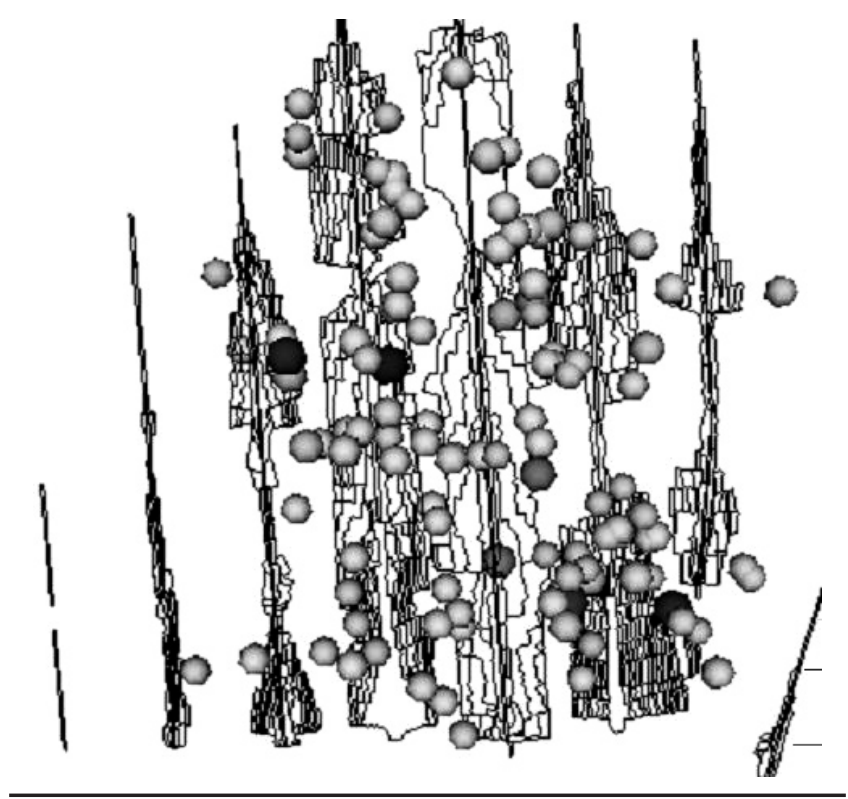

FIG. 4 Mining and seismicity $(M>\approx 2)$

\subsection{Selection of Seismic Events}

The mine catalogue included numerous small events that were associated with off-reef development (footwall haulages and cross cuts to reef). Richardson and Jordan (2002) showed that events associated with advancing development had Moment-Magnitude $\mathrm{M}(\mathrm{Mo})<0.5$ in a study of nearby mines. They labelled these events type " $\mathrm{A}$ " events and interpreted them as "fracture-dominated" rupture events, as against the normal type " $\mathrm{B}$ " "friction-dominated" slip events. We presented several lines of evidence in Cichowicz et al. (2004) that these events were the actual development blasts. They will be called "blasts" in this study.

As this present study relates to seismicity induced by stoping and not by tunnel development, we need to exclude these blast events from the analysis. The simplest way to exclude development events would be to exclude all events with $\mathrm{M}(\mathrm{Mo})<0.5$. This would unfortunately involve excluding in excess of $80 \%$ of the recorded events.

Following the suggestion of Richardson and Jordan (2002), the development blast events were identified as those with $\mathrm{M}(\mathrm{Mo})<0.5$ and that located within $100 \mathrm{~m}$ of another events and within 80 s of another event. 
TABLE 3 Number of blast and induced events greater than three values of Moment-Magnitudes

\begin{tabular}{llll}
\hline $\mathbf{N}$ & $\mathbf{M}>-\mathbf{0 . 5}$ & $\mathbf{M}>\mathbf{0 . 0}$ & $\mathbf{M}>\mathbf{0 . 5}$ \\
\hline Blast & 26425 & 5652 & 49 \\
Induced & 17082 & 12198 & 7284 \\
Total & 43507 & 17850 & 7333 \\
\hline
\end{tabular}

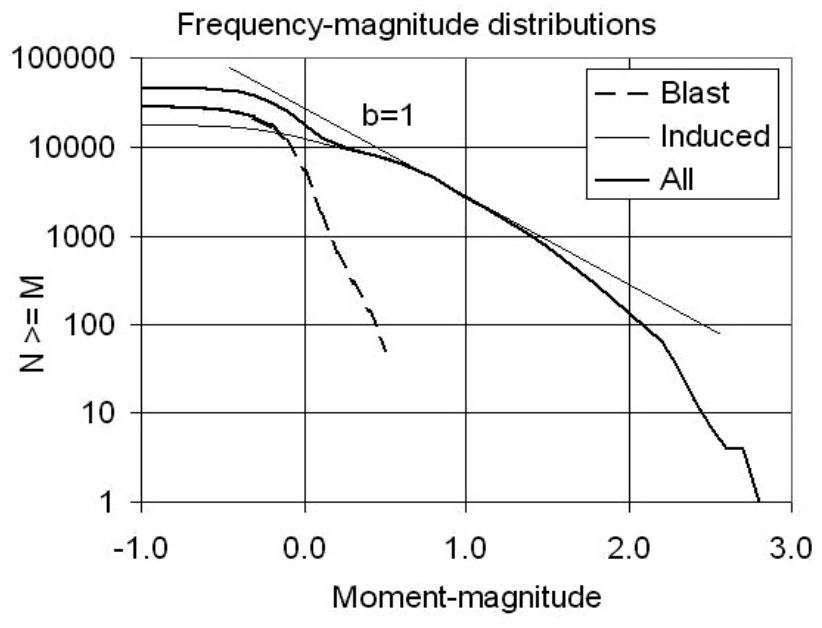

FIG. 5 Size distribution of events identified as blast events, mining-induced events and all events

This division of events into "blast" and "induced" events is shown in Table 3 and Figure 5. An increasing proportion of all events are likely to have been blast events as the threshold magnitude drops below 0.5 . In the process, the "hump" in the frequency-magnitude distribution in the range $0.0<\mathrm{M}<0.5$ that is visible in the graph for the entire data set disappears.

The 12198 events with $M>0.0$ that were most likely to be induced events are used in this study.

\subsection{Identification of "Working Areas"}

The methodology outlined above was applied to the Driefontein data set. An example of mining and seismicity within part of the area during a single time step is shown in Figure 6. Three distinct regions of active mining can be identified. These will be termed "working areas". In the normal mining operation these working areas would probably be lumped together into a single "polygon" for seismic analysis to avoid misallocating events into the wrong polygon. In this analysis, the influence of events that locate between the working areas, such as A, B and C in Figure 6 , are split between these areas according to their proximity to current mining.

An algorithm was written to identify such regions for each mining step. This algorithm was based on their being separated by at least three elements that were not being mined at that time.

\subsection{Analysis of Predictions using the Three Methods}

Observed seismicity is compared to seismicity predicted using the three methods described above. Data for the 99 working areas that included at least 10 mined elements are now shown in Figures 7, 8 and 9.

In Figure 7, the total observed seismic moment in each region is compared to the seismic moment predicted using the integration model. Note the dominant contribution of the few largest events, which appear as unpredicted

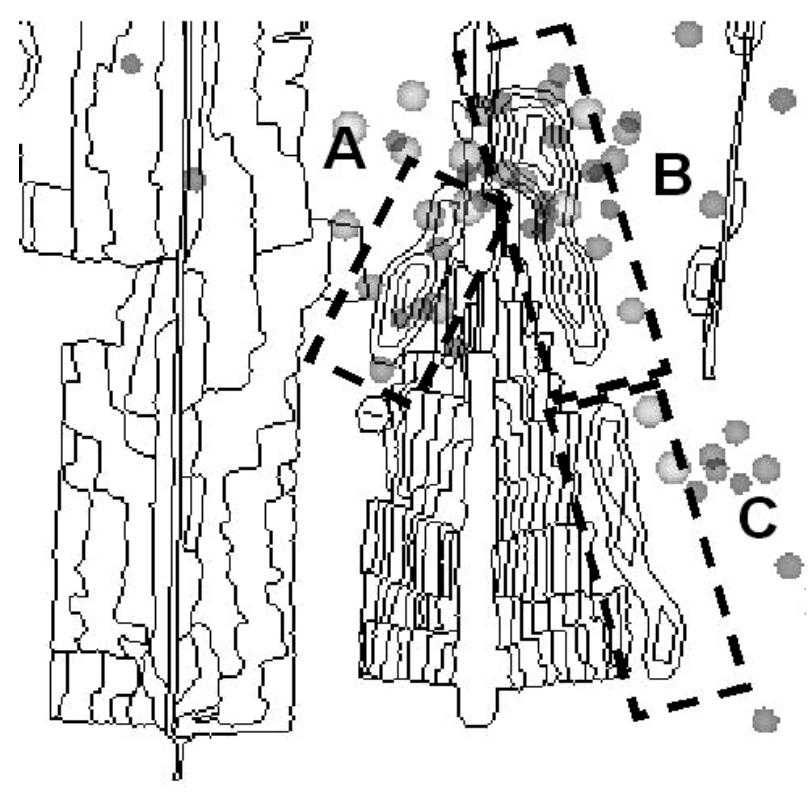

FIG. 6 An example of extraction in the three months from November 2001 shown as contours, the larger seismic events as opaque balls and mining steps up to August 2001 as outlines

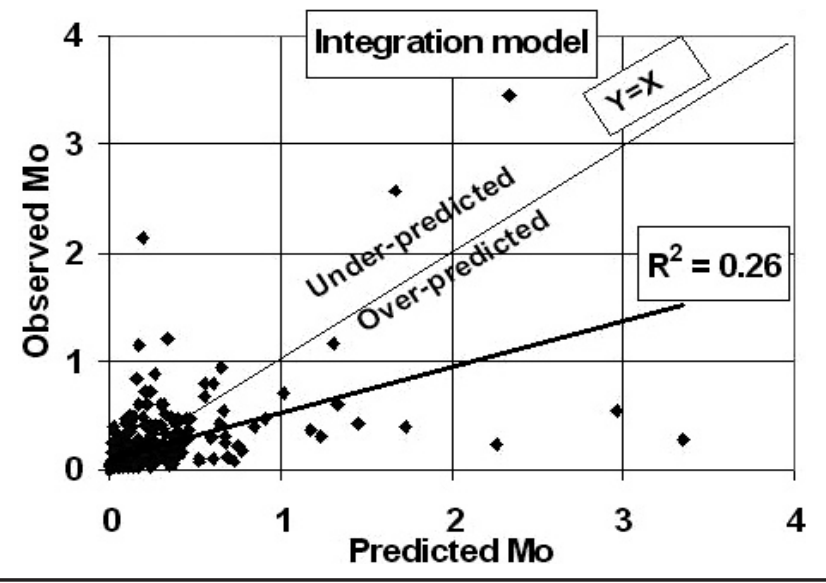

FIG. 7 Predicted and observed seismic moment, GN-m per grid element

(labelled "under-predicted": $\mathrm{Y}>\mathrm{X}$ ) or as over-predicted $(\mathrm{Y}<\mathrm{X})$ depending whether the large events are to be predicted or are "current" events.

Using the number of events eliminates any dominance of the effect of any event on any other. Figure 8 shows the comparison between predicted and observed number of seismicity per area mined. An inspection of this Figure shows, inter alia, that:

a) Although the correlation factor $\left(\mathrm{R}^{2}\right.$ value $)$ is well below 0.5 , this is a reflection of the detail of the data as most of the more active working areas, in which more than two events per element occurred, were predicted as being more likely to be seismically active.

b) The most noticeable feature in this figure is the fact that almost all predictions were under-estimates, in other words the seismic event rate almost always increased with time in any working area. This occurred because the span and therefore the face stresses and values of ERR increased. 
c) The data distribution shown in Figure $8 \mathrm{c}$ is similar to that shown in Fig. 8b. The data are about evenly spread across the "perfect" line, labelled " $Y=X$ ". This means that the ERR correction implied by Equation [7] provided, on average, an appropriate correction for the deficit apparent in Figure 8b.

If this approach were to be used for deciding whether to continue mining in an area or not, the bounding line labelled $\mathrm{Y}=\mathrm{X}+1$ provides a conservative upper limit to the impending seismicity. Working places that were predicted to result in less than one event per element did indeed generate this small seismicity rate.

The data points within the dashed ellipse in Figure 8c were the most severe examples of over-prediction. This will be contrasted later with a similar region in Figure 9c.

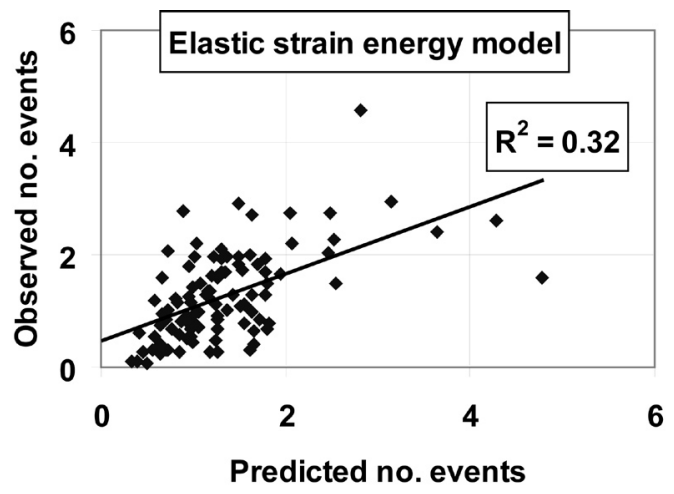

(a)

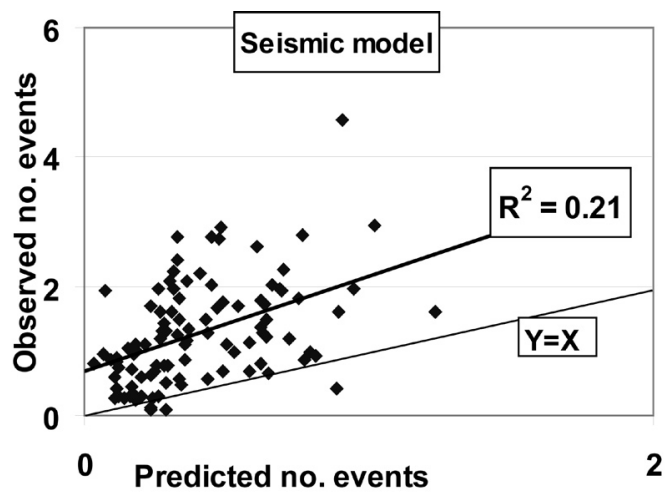

(b)

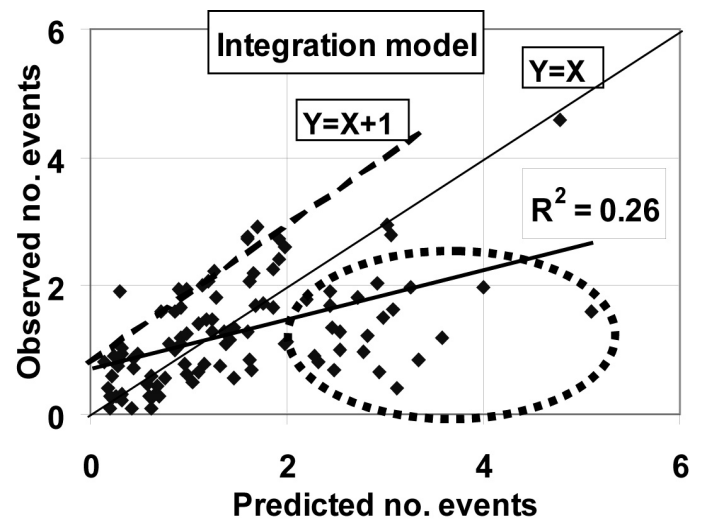

(c)

FIG. 8 Comparison of the observed number of events per mined element with the predicted number of elements by each of three model approaches. The ellipse in (c) is drawn around some of the data that were over-predicted
Predicting the number of events is of limited value because most of these events are of negative magnitude and do not cause any damage. As shown in Figure 7 the opposite extreme of using seismic moment to predict the amount of seismic moment is ineffective. We now compromise by measuring each seismic event as source radius multiplied by apparent stress $\left(r_{0} \times \tau\right)$, labelled "rad_tau" in Figure 9. This parameter was chosen in an attempt to represent both the extent $\left(\mathrm{r}_{0}\right)$ and the intensity $(\tau)$ of strong ground motion.

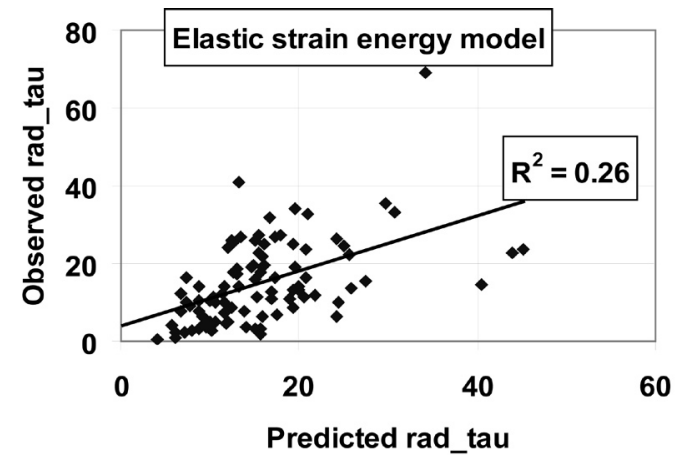

(a)

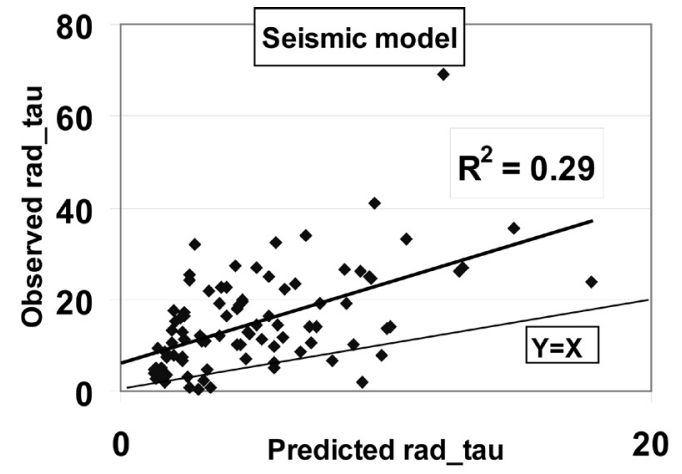

(b)

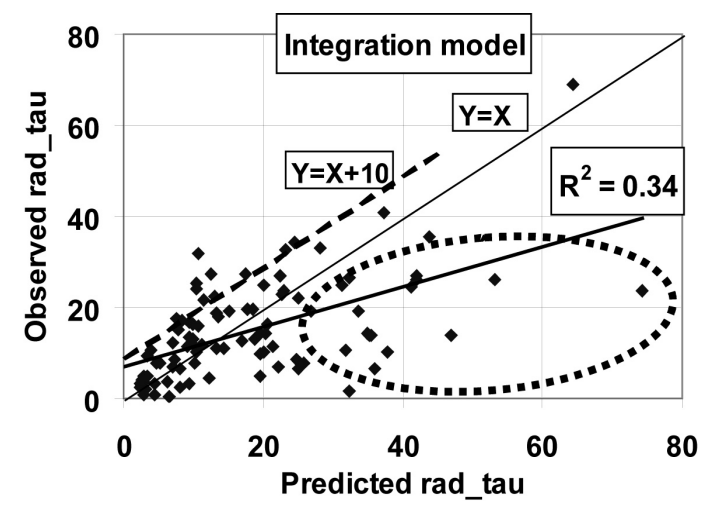

(c)

FIG. 9 Comparison between the observed number of events per mined element and source radius time apparent stress, MPa-m

The small weighting given to larger events by applying a "strength" of $r_{0} \times \tau$ gives better results for the seismicity-related predictors, as seen in Figure $9 \mathrm{~b}$ and c. In particular, the number of working places that had their seismicity severely over-predicted decreased, as can been by fewer events falling in the elliptical region of Figure 9c compared to the similar region in Figure 8c. 


\section{CONCLUSIONS AND DISCUSSION}

This paper describes the principles of a methodology and makes reference to a computer program for an integrated analysis of both rockmass modelling and observed seismicity to predict, or forecast, the amount of seismicity that will be likely to occur over the next month or quarter in any working area.

The results show the potential benefits of this type of integration work for mine layout planning. The techniques presented here for associating observed seismicity with modelled seismicity are still very new and further developments of the method and more detailed analysis will undoubtedly lead to better interpretation of the likely response, in time and space, of seismicity to mining.

Although the results are not sufficiently convincing to be implemented, they are encouraging. As this is the first analysis using this methodology, there will obviously be much scope for improvement, even in the short term. A partial list would include:

- Modelling. There are a number of ways of introducing easy approximations to simulate actual rock failure (e.g. Spottiswoode, 2001). These should provide better insights into the rockmass response at high levels of ERR and over time.

- Seismic data. Seismic locations can be improved using methods described in Cichowicz (2004).

- Statistical processing. Very simple methods have been used in this study. For example, all working areas have been given equal weight and equal symbol sizes in Figures 7 to 9, even though the larger areas behaved better. Improved methods will be essential before this work can be used for quantitative hazard analysis.

\section{ACKNOWLEDGMENTS}

This work was supported by SIMRAC under projects SIM 02-03-04 and SIM 04-03-01. Thanks to Driefontein Consolidated for permission to use their data. Members of the SIMRAC Rock Engineering expert committee encouraged me to move forward with this predictive model. Discussions with and reviews by Ray Durrheim and Mark Hildyard were essential in keeping this work reasonably comprehensible, as were comments from an anonymous referee.

\section{REFERENCES}

Beck, D.A. and Brady, B.H.G. (2001) A numerical method for engineering management of induced seismic risk in hard rock mining. In 5th Int Symposium on Rockbursts and Seismicity in Mines, S. A. Inst. Min. Metall., pp. 457-463.

Cichowicz, A., Spottiswoode, S.M., Linzer, L.M., Heyns, S.P., Drent, D. and Handley, M.F. (2004) Improved seismic locations and location techniques. SIMRAC final report in preparation for SIM020304.

Côté, M., Mitchelson, C. and Alcott, J.M. (2001) Seismic monitoring and numerical modelling decision-making at McWatters' Sigma Mine. In 5th Intl Symposium on Rockbursts and Seismicity in Mines, S. A. Inst. Min. Metall., pp. 427-431.

Handley, M.F., de Lange, J.A.J., Essrich, F. and Banning, J.A. (2000) A review of the sequential grid mining method employed at Elandsrand Gold Mine. J. S. Afr. Inst. Min. Metall., pp. 1183-1194.

Hoffmann, G., Sewjee, R. and van Aswegen, G. (2001) First steps in the integration of numerical modelling and seismic monitoring. In 5th Int Symposium on Rockbursts and Seismicity in Mines, S. A. Inst. Min. Metall., pp. 397-404.

Kijko, A., Lasocki, A. and Graham, G. (1998) Quantification of Seismic Hazard from Seismic Events in Mines. SIMRAC GAP517.

Klokow, J.W., Riemer, K.L and Ferreira, R.I. (2003) An initial assessment of the closely spaced dip -pillar mining layout as practiced at Driefontein Gold Mine. In ISRM 2003- Technology roadmap for rock mechanics, S. A. Inst. Min. Metall., pp. 655-663.

Lachenicht, R., Wiles, T. and van Aswegen, G. (2001) Integration of deterministic modelling with seismic monitoring for the assessmen of the rockmass response to mining: Part II Applications In 5th Intl Symposium on Rockbursts and Seismicity in Mines, S. A. Inst. Min. Metall., pp. 389-395.

Richardson, E. and Jordan, T.J. (2002) Seismicity in deep gold mines of South Africa: Implications for tectonic earthquakes, Bull. Seism. Soc. Amer, 92: pp. 1766-1782.

Ryder, J.A and Jager, A.J. editors (2002) A textbook on Rock Mechanics for tabular hard rock mines. 1st Edition, SIMRAC: Johannesburg.

Spottiswoode, S.M. and de Beer, W. (1999) A review of current seismic prediction and hazard assessment practice and capability, Final Report for Deepmine Project No 5.4.1.

Spottiswoode, S.M. (2001) Keynote address: Synthetic seismicity mimics observed seismicity in deep tabular mines. In 5th Intl Symposium on Rockbursts and Seismicity in Mines, S. Afr. Inst. Min. Metall., pp. 371-378.

Spottiswoode, S.M. (2004) A seismic and modelling analysis of dip-pillar mining. 2nd International Seminar on Deep and High Stress Mining, S. Afr. Inst. of Min. Metall., pp. 67-78.

van Antwerpen, H.E.F. and Spengler, M.G. (1982) The effect of mining-related seismicity on excavations at East Rand Propriety Mines, Limited. In 1st Intl. Symposium on Rockbursts and Seismicity in Mines, Johannesburg, S. Afr. Inst. of Min. Metall : pp. 29-37.

Wiles, T., Lachenicht, R. and van Aswegen, G. (2001) Integration of deterministic modelling with seismic monitoring for the assessment of the rockmass response to mining: Part 1 Theory In 5th Intl Symposium on Rockbursts and Seismicity in Mines, S. A. Inst. Min. Metall., pp. 379-387. 